\title{
ESTENOSE DEGENERATIVA DO CANAL LOMBAR: CORRELAÇÃO ENTRE O ÍNDICE DE OSWESTRY E IMAGEM DE RESSONÂNCIA MAGNÉTICA
}

\author{
DEGENERATIVE LUMBAR SPINAL STENOSIS: CORRELATION BETWEEN OSWESTRY \\ INDEX AND MAGNETIC RESONANCE IMAGING
}

\section{ESTENOSIS DEGENERATIVA DEL CANAL LUMBAR: CORRELACIÓN ENTRE EL ÍNDICE DE OSWESTRY Y LA RESONANCIA MAGNÉTICA}

Wagner Pasoualini ${ }^{1}$, Marcelo Ítalo Risso ${ }^{1}$, Guilherme Rebechi Zuiani ${ }^{1}$, João Batista de Miranda ${ }^{2}$

\begin{abstract}
RESUMO
Objetivos: Verificar a relação entre o grau de estenose do canal lombar (ECL) com a ressonância nuclear magnética (RM) e a gravidade da disfunção, de acordo com o Índice de Oswestry em pacientes com ECL e em controles. Métodos: Vinte e três pacientes com diagnóstico de ECL foram comparados com grupo controle de 17 voluntários. Todos foram submetidos a RM e responderam ao questionário de Oswestry. A análise estatística utilizou o teste exato de Fisher, de Mann-Whitney e de Spearman. Resultados: Dor lombar foi a queixa mais frequente em ambos os grupos. O índice de Oswestry mostrou disfunção, em média, em 45,69\% no grupo ECL e 11,60\% no controle. A RM revelou que a área longitudinal do saco dural, o diâmetro do canal e os recessos laterais e forames estavam igualmente alterados em ambos os grupos. Conclusões: Não houve correlação entre o grau de estenose e o índice de Oswestry nos dois grupos.
\end{abstract}

Descritores: Estenose espinal; Imagem por ressonância magnética; Avaliação da deficiência; Dor nas costas; Dor lombar.

\begin{abstract}
Objectives: To verify the relationship between the degree of lumbar spinal canal stenosis (LCS), in magnetic resonance imaging (MRI) and the severity of disability according to the Oswestry Index in patients with LCS compared to controls without LCS. Methods: Twenty-three patients with a diagnosis of LCS were compared with a control group of 17 volunteers. All participants underwent MRI and completed the Oswestry questionnaire. Statistical analysis used the Fisher exact test, the Mann-Whitney and the Spearman's tests. Results: Low back pain was the most frequent complaint in both groups. The Oswestry index showed average disability in $45.69 \%$ patients and $11.60 \%$ controls. $M R I$ revealed that the dural sac cross-sectional area, the diameter of the canal and the lateral recesses and foramina were equally changed in both groups. Conclusions: There was no correlation between the degree of lumbar canal stenosis and the disability index in both groups.
\end{abstract}

Keywords: Spinal stenosis; Magnetic resonance imaging; Disability evaluation; Back pain; Low back pain.

\section{RESUMEN}

Objetivos: Investigar la relación entre el grado de estenosis de canal lumbar (ECL) con imágenes de resonancia magnética (RM) y la gravedad de la disfunción de acuerdo con el Índice de Oswestry en pacientes con ECL y controles. Métodos: Veintitrés pacientes con un diagnóstico de ECL se compararon con un grupo control de 17 voluntarios. Todos los pacientes fueron sometidos a resonancia magnética y completaron el cuestionario Oswestry. Para el análisis estadístico se utilizó la prueba exacta de Fisher, Mann-Whitney y de Spearman. Resultados: El dolor de espalda es la queja más frecuente en ambos grupos. El índice de disfunción de Oswestry mostró en promedio 45,69\% para el grupo ECL y 11,60\% para el grupo control. La RM demostró que el área longitudinal del saco dural, el diámetro del canal y de los agujeros y las cavidades laterales se han cambiado de manera similar en ambos grupos. Conclusiones: No se encontró correlación entre el grado de estenosis y el índice de Oswestry en ambos grupos.

Descriptores: Estenosis espinal; Imagen por resonancia magnética; Evaluación de la discapacidad; Dolor de espalda; Dolor de la región lumbar.

\section{INTRODUÇÃO}

A estenose do canal lombar (ECL) é uma doença cada vez mais frequente e está relacionada à expectativa de vida maior da população ${ }^{1}$. O estreitamento anormal do canal vertebral leva a sintomatologia local ou a distância, relacionada à compressão das raízes nervosas. Esse estreitamento ocorre devido a doenças congênitas ou degenerativas ${ }^{2}$. Na doença degenerativa, ocorre comprometimento do disco intervertebral, formação de osteófitos, hipertrofia facetária e ligamentar, levando este conjunto de alterações a uma diminuição do diâmetro anteroposterior e transverso do canal lombar ${ }^{3}$.
O diagnóstico da ECL é frequentemente tardio. A doença ocorre de forma insidiosa, com períodos de piora e melhora, com evolução lenta, associada a comorbidades que aparecem no envelhecimento da população, o que dificulta o diagnóstico diferencial ${ }^{4}$. Apesar de todos os sintomas, os sinais clínicos são escassos ${ }^{5}$.

Três síndromes neurológicas estão relacionadas à ECL:6

- Síndrome de claudicação neurológica: paciente apresenta dor em membros inferiores apenas à deambulação;

- Síndrome radicular: paciente apresenta dor em membros inferiores quando em pé ou sentando, podendo melhorar com repouso;

1. Médico Assistente do Grupo de Cirurgia da Coluna, Universidade Estadual de Campinas - Unicamp, Campinas, SP, Brasil.

2. Professor Assistente, Doutor do Departamento de Ortopedia eTraumatologia da Universidade Estadual de Campinas - Unicamp, Campinas, SP, Brasil.

Trabalho realizado no Departamento de Ortopedia eTraumatologia, Grupo de Cirurgia da Coluna, Universidade Estadual de Campinas (Unicamp).Brasil. Correspondência: Rua Joaquim Pires de Oliveira, 176, Jundiaí, SP, Brasil. 13201-847. Email: wagnerpas1@uol.com.br

Recebido em 25/05/2012, aceito em 08/08/2012 
- Síndrome mista: dor em membros inferiores que piora com a deambulação.

Com a evolução dos estudos de imagem, o diagnóstico de ECL tornou-se mais frequente e preciso ${ }^{7}$. À suspeita de ECL, exames de imagem são indicados, sendo a ressonância magnética (RM) o exame de escolha, com alta sensibilidade ${ }^{8}$.

A medida da área de secção transversal do saco dural (ASTSD) e o diâmetro anteroposterior são utilizadas para diagnóstico de estenose central do canal lombar',10. São considerados, para o diagnóstico de estenose lateral, a altura e a profundidade do recesso lateral e, para a estenose foraminal, o diâmetro do forame ${ }^{11}$.

Embora o estudo de imagem através da RM tenha contribuído para a melhor avaliação do canal vertebral, com a medida da área e do diâmetro do canal, o grau de estreitamento do canal vertebral que causa sintoma permanece duvidoso ou conflitante na literatura ${ }^{11,12}$. A avaliação do paciente tem sido subjetiva e variável, e o critério diagnóstico ainda não foi bem normatizado ${ }^{13}$. Sendo assim, é muito importante que sejam realizados estudos que correlacionem o nível de incapacidade dos pacientes com a estenose do canal vertebral comparando com os achados de exames de imagem.

O índice de Oswestry (ODI) é um questionário auto-preenchido pelo paciente, que analisa os níveis de percepção de incapacidade em 10 atividades cotidianas de vida diária, para atribuir uma pontuação subjetiva de nível de função. O questionário tem como vantagem ser de fácil compreensão, e engloba um amplo domínio da dor, função e limitação do estado de saúde ${ }^{14,15}$.

O objetivo deste estudo foi estabelecer a relação existente entre o grau de ECL, observado em exame de RM, com o índice de Oswestry em pacientes com ECL, fazendo a comparação com um grupo controle de indivíduos que comprovadamente não apresentam estenose.

\section{MÉTODOS}

Este é um estudo transversal, caso-controle, realizado no Ambulatório de Coluna do Hospital de Clínicas da Universidade Estadual de Campinas (Unicamp), de atendimento público, no período de julho de 2010 a outubro de 2011. Todos os pacientes foram encaminhados ao Hospital para tratamento cirúrgico e somente os que tiveram o diagnóstico de estenose lombar e indicação cirúrgica confirmados através da anamnese, exame físico e exame de ressonância magnética (RM), avaliados por pelo menos dois cirurgiões de coluna do serviço, foram incluídos neste estudo. Este grupo com 23 pacientes com mais de 40 anos, foi denominado Grupo Estenose. A indicação para cirurgia dos pacientes com estenose verificada à RM foi feita com base na queixa principal do paciente, podendo ser lombalgia mais radiculopatia ou claudicação neurogênica.

O Grupo Controle com 17 indivíduos foi recrutado por meio da indicação dos pacientes do Grupo Estenose, ou seja, solicitou-se que cada paciente do Grupo Estenose indicasse um indivíduo com 40 anos ou mais, sadio e sem diagnóstico conhecido de afecção na coluna, independentemente do sexo, e disposto a participar do estudo, realizando exame de RM e respondendo ao questionário de Oswestry.

Os critérios de inclusão para o Grupo Estenose foram: presença de estenose do canal lombar, diagnosticada através de história clínica e confirmada através de ressonância magnética.

Os critérios de exclusão foram: hérnia do disco lombar isolada, cirurgia prévia da coluna, neuropatia periférica, vasculopatia, diabetes e alcoolismo.

No Grupo Controle o critério de inclusão foi a ausência de patologia na coluna. Os critérios de exclusão foram: cirurgia prévia da coluna, neuropatia periférica, vasculopatias, diabetes e alcoolismo.

O estudo foi aprovado pelo Comitê de Ética da Faculdade de Ciências Médicas da Unicamp (processo numero 959/2007) e o consentimento informado foi obtido de todos os participantes.

À anamnese, os sintomas analisados foram: lombalgia, radiculopatia e claudicação neurogênica. Devido à possibilidade de um indivíduo ter mais de uma queixa, aquela mais importante para o paciente foi nomeada como principal, e a segunda como secundária. Registrou-se "queixa mista" quando o paciente apresentava as três queixas. Neste estudo, analisou-se a correlação entre as queixas lombalgia, radiculopatia, claudicação neurogênica e queixa mista com o grau de estenose verificado no exame de RM.

Todos os indivíduos de ambos os grupos foram submetidos a exame de RM de 3,0T (Philips modelo Achieva) no mesmo serviço. Os participantes foram colocados em decúbito dorsal e foi utilizado protocolo padrão para realização do exame em todos os indivíduos.

Os dados da digitalização da RM com imagens ponderadas em T2 foram inseridos em computador para cálculo da área de secção transversa do saco dural (ASTSC) e do diâmetro anteroposterior. Ambos foram medidos digitalmente na parte central do disco de L1 a S1, utilizando o programa Osirix 4.0 para Macintosh. A ASTSC superior a $100 \mathrm{~mm}^{2}$ foi considerada medida normal; 76-99 mm², considerada como indicativa de estenose moderada e inferior a $76 \mathrm{~mm}^{2}$, como evidência de estenose grave. O comprometimento da raiz nervosa no forame foi classificada como segue: grau 0, nenhum contato da raiz com o disco; grau 1, contato com a raiz sem desvio; grau 2, contato com desvio da raiz e grau 3, compressão da raiz com deformação ${ }^{16}$.

Os participantes do estudo foram orientados a responder e completar a tradução nacional validada do questionário de incapacidade de Oswestry ${ }^{17}$. A orientação para preenchimento do questionário foi feita por pesquisador diferente daquele que fez a anamnese. O questionário contém seis afirmações (níveis denotando 0 a 5) em cada uma das 10 seções, relacionadas com deficiências como a dor, e habilidades, tais como cuidados pessoais, levantar, andar, sentar, dormir, vida sexual, vida social e viajar. Em cada seção, o paciente escolhe a afirmação que melhor descreve a sua ou seu status. As respostas escolhidas receberam pontuação de 0 a 5. A pontuação total poderia, portanto, variar de zero (o mais alto nível de função) a 50 (nível mais baixo de função). Após somar todos os pontos, a pontuação total foi dividida por 50 e multiplicada por 100 para calcular o percentual de invalidez.

\section{ANÁLISE ESTATÍSTICA}

Medidas qualitativas foram descritas segundo grupos com uso de frequências absolutas e relativas e foi verificada a existência de associação dessas medidas com os grupos com uso do teste exato de Fisher ${ }^{18}$. As medidas quantitativas foram descritas segundo grupos com uso de medidas resumo (média, desvio padrão, mediana, mínimo e máximo) e comparadas entre os grupos com o uso do teste Mann-Whitney ${ }^{18}$. Para verificar a existência de relação das medidas quantitativas e gradativas da RM com as medidas quantitativas clínicas e de função dos pacientes, foram calculadas as correlações de Spearman ${ }^{18}$ entre elas. Os testes foram realizados com nível de significância de 5\%.

\section{RESULTADOS}

Os grupos eram homogêneos quanto à idade e sexo. No Grupo Estenose, a idade variou de 40 a 78 anos, média de 55 anos com desvio padrão de $11,93 \%$. No Grupo Controle a idade variou entre 41 e 71 anos, média de 51,3 anos e desvio padrão de 9,52\%. Não houve diferença significativa entre os grupos. No Grupo Estenose, houve predomínio do sexo masculino, enquanto que no Grupo Controle, houve predomínio do sexo feminino, sem diferença significativa entre os grupos.

A lombalgia esteve presente em ambos os grupos. Foi verificada como queixa primária em 47,6\% dos pacientes do Grupo Estenose e $21,4 \%$ dos participantes do Grupo Controle, sem diferença significativa para a queixa de lombalgia e as demais queixas entre os grupos (Figura 1). Como queixa secundária, a lombalgia apresentou maior frequência no Grupo Estenose ( $p=0,005)$ (Figura 2).

Quanto as demais queixas, primária ou secundária, não houve diferenças estatísticas significantes entre os grupos (Tabela 1).

$\mathrm{Na}$ avaliação dos resultados do índice de Oswestry, encontramos que 32 pacientes completaram o questionário, sendo 17 do Grupo Estenose e 15 no Grupo Controle (os dados dos 


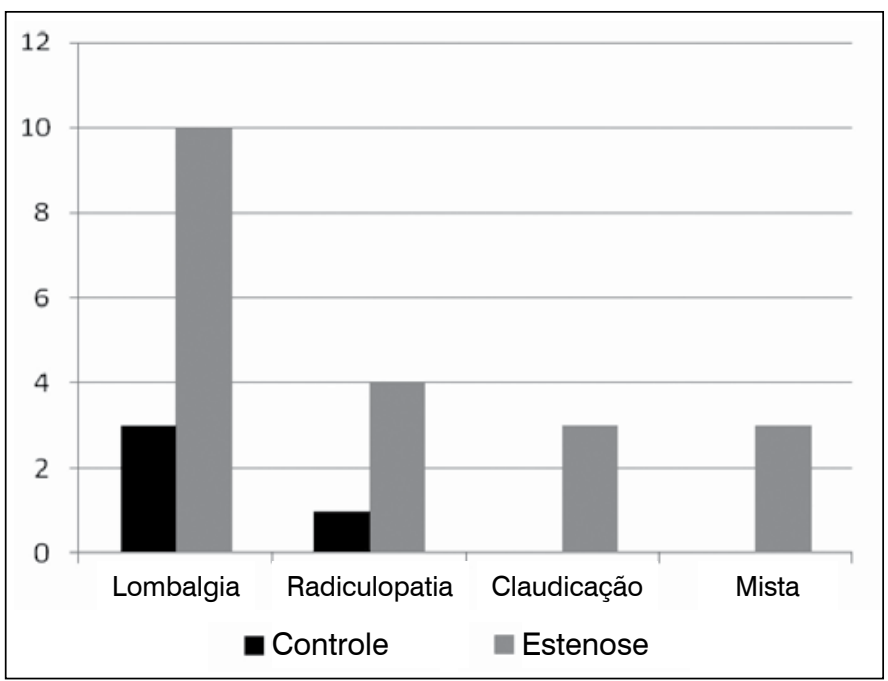

Figura 1. Representação gráfica da queixa primária apresentada pelos pacientes em ambos os grupos.

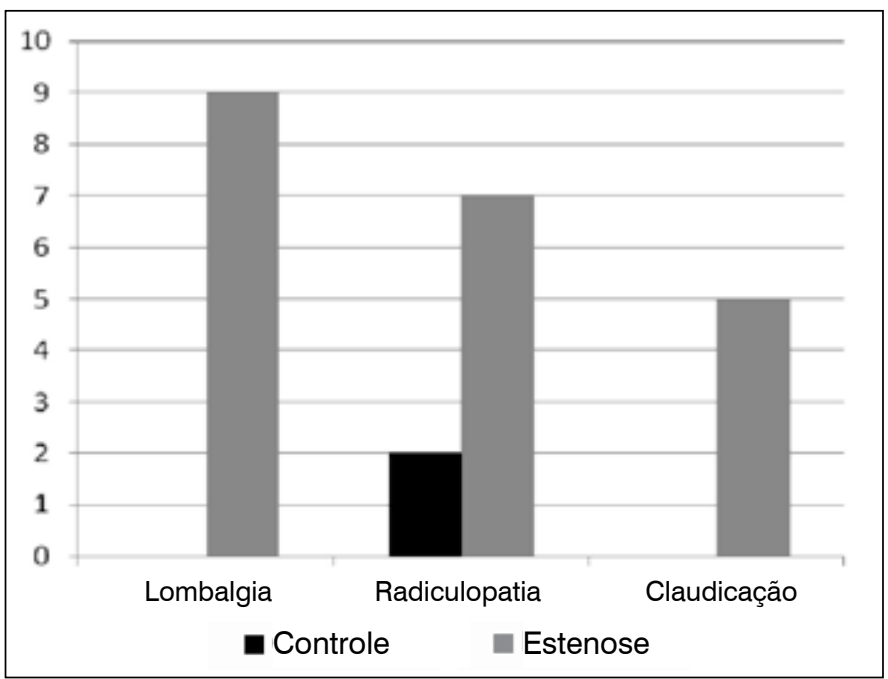

Figura 2. Representação gráfica da queixa secundária apresentada pelos pacientes em ambos os grupos.

Tabela 1. Variáveis clínicas de acordo com os grupos e resultados dos testes estatísticos.

\begin{tabular}{|c|c|c|c|c|c|c|c|}
\hline \multirow[b]{2}{*}{ Variável } & \multicolumn{4}{|c|}{ Grupo } & & & \\
\hline & \multicolumn{2}{|c|}{ Controle } & \multicolumn{2}{|c|}{ Estenose } & \multicolumn{2}{|c|}{ Total } & $p$ \\
\hline & $n$ & $\%$ & $n$ & $\%$ & $\mathrm{n}$ & $\%$ & \\
\hline
\end{tabular}

Queixa principal

\begin{tabular}{c|c|c|c|c|c|c|c}
\hline Lombalgia & 3 & 21,4 & 10 & 47,6 & 13 & 37,1 & 0,162 \\
\hline Radiculopatia & 1 & 7,1 & 4 & 19,0 & 5 & 14,3 & 0,627 \\
\hline Claudicação & 0 & 0,0 & 3 & 14,3 & 3 & 8,6 & 0,259 \\
\hline Queixa mista & 0 & 0,0 & 3 & 14,3 & 3 & 8,6 & 0,259 \\
\hline
\end{tabular}

Queixa secundária

\begin{tabular}{c|c|c|c|c|c|c|c}
\hline Lombalgia & 0 & 0,0 & 9 & 42,9 & 9 & 25,7 & 0,005 \\
\hline Radiculopatia & 2 & 14,3 & 7 & 33,3 & 9 & 25,7 & 0,262 \\
\hline Claudicação & 0 & 0,0 & 5 & 23,8 & 5 & 14,3 & 0,069 \\
\hline
\end{tabular}

questionários de 8 participantes estavam incompletos e foram desconsiderados para este estudo). No Grupo Estenose, encontramos o percentual de incapacidade, ou seja, escore de, mínimo de $20 \%$ e máximo de $68 \%$ (média 45,59\%), com desvio padrão de 17,39\%; no Grupo Controle, encontramos mínimo de 0 e máximo de 68\% de percentual de incapacidade (média de 11,80\%), com desvio padrão de 17,88\% Houve uma diferença significativa ( $p<0,001$ ) entre os dois grupos.

A RM foi realizada do segmento de L1 a S1. Foi possível medir a ASTSD e o diâmetro do canal em 173 níveis, sendo 108 do Grupo Estenose e 65 do Grupo Controle. Na avaliação dos resultados, os pacientes do grupo Estenose apresentam menores valores de ASTSD nos níveis L3-L4 ( $p=0,007)$, L4-L5 ( $p=<0,001)$ e L5-S1 ( $p=0,011)$ quando comparados com os do Grupo Controle (Figura 3). O diâmetro do canal mostrou-se menor em todas as vértebras do Grupo Estenose ( $p<0,001$; Figura 4). Não houve correlação estatística entre o grau de estenose e os sintomas de dor, radiculopatia e claudicação

Foi possível avaliar pela RNM um total de 386 recessos laterais e forame 226 do Grupo Estenose e 160 do Grupo Controle. Deste total, $141(62,4 \%)$ raízes não tiveram qualquer contato com o disco adjacente no Grupo Estenose e 141 (88\%) no Grupo Controle; contato com a raiz, 31 (13,7\%) no Grupo Estenose e 12 (7,5\%) no Grupo Controle; contato e desvio da raiz, 24 (10,6\%) no Grupo Estenose e 4 (2,5\%) no Grupo Controle; compressão da raiz com deformação, 30 (13,2\%) Grupo Estenose e 3 (1,8\%) no Grupo Controle. O contato com a raiz em qualquer grau mostrou-se ligeiramente superior no grupo com estenose do canal, sendo a diferença significativa à direita em L5-S1 $(p=0,050)$ e à esquerda em L4-L5 ( $p<0,001)$ e L5-S1 ( $p=0,022)$ (Tabela 2).

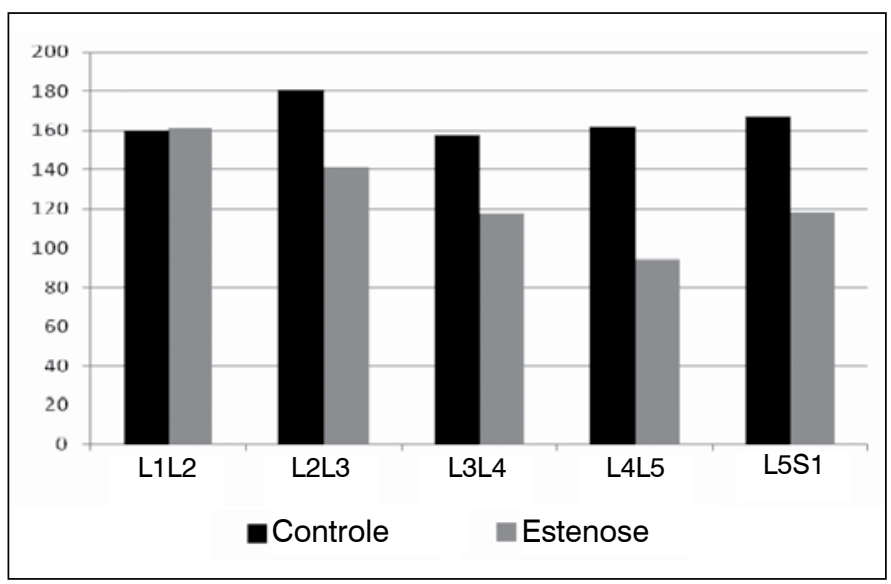

Figura 3. Representação gráfica da área de secção transversa do saco dural na RM.

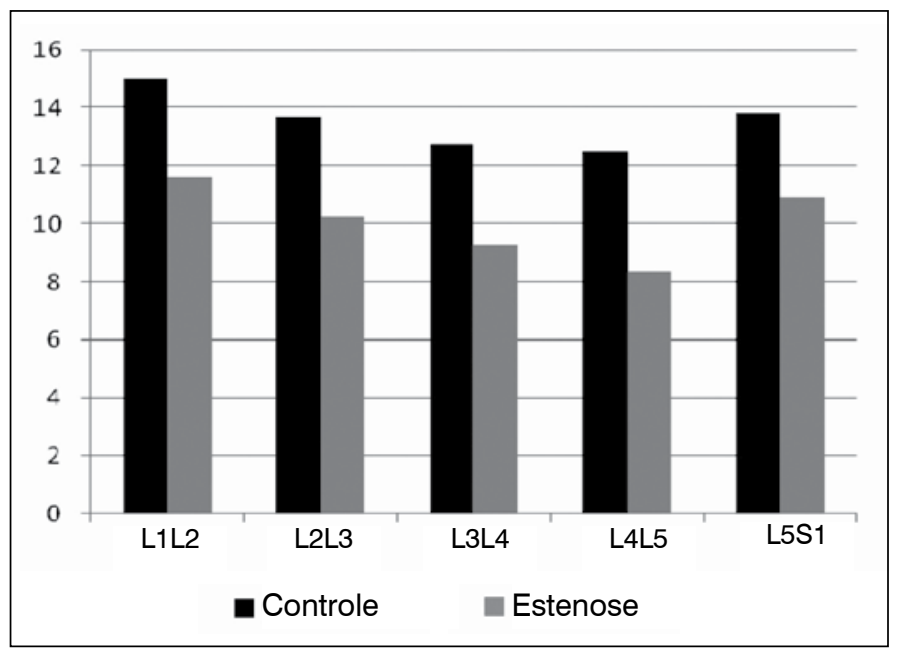

Figura 4. Representação gráfica da medida do diâmetro do canal na RM. 
Tabela 2. Medidas do forame e resultados dos testes estatísticos.

\begin{tabular}{|c|c|c|c|c|c|c|c|c|}
\hline \multirow{2}{*}{ Nível } & \multirow{2}{*}{ Categoria } & \multicolumn{2}{|c|}{ Grupo Controle } & \multicolumn{2}{|c|}{ Grupo Estenose } & \multicolumn{2}{|c|}{ Total } & \multirow{2}{*}{$\mathrm{p}$} \\
\hline & & $\mathrm{n}$ & $\%$ & $\mathrm{n}$ & $\%$ & $\mathrm{n}$ & $\%$ & \\
\hline \multicolumn{9}{|c|}{ Forame (direita) } \\
\hline \multirow{2}{*}{ L1-L2 } & 0 & 16 & 100,0 & 21 & 95,5 & 37 & 97,4 & \multirow{2}{*}{0,827} \\
\hline & 3 & 0 & 0,0 & 1 & 4,5 & 1 & 2,6 & \\
\hline \multirow{4}{*}{ L2-L3 } & 0 & 15 & 93,8 & 18 & 81,8 & 33 & 86,8 & \multirow{4}{*}{0,569} \\
\hline & 1 & 0 & 0,0 & 3 & 13,6 & 3 & 7,9 & \\
\hline & 2 & 1 & 6,3 & 0 & 0,0 & 1 & 2,6 & \\
\hline & 3 & 0 & 0,0 & 1 & 4,5 & 1 & 2,6 & \\
\hline \multirow{4}{*}{ L3-L4 } & 0 & 14 & 87,5 & 12 & 52,2 & 26 & 66,7 & \multirow{4}{*}{0,069} \\
\hline & 1 & 1 & 6,3 & 6 & 26,1 & 7 & 17,9 & \\
\hline & 2 & 0 & 0,0 & 1 & 4,3 & 1 & 2,6 & \\
\hline & 3 & 1 & 6,3 & 4 & 17,4 & 5 & 12,8 & \\
\hline \multirow{4}{*}{ L4-L5 } & 0 & 12 & 75,0 & 15 & 65,2 & 27 & 69,2 & \multirow{4}{*}{0,489} \\
\hline & 1 & 3 & 18,8 & 2 & 8,7 & 5 & 12,8 & \\
\hline & 2 & 0 & 0,0 & 3 & 13,0 & 3 & 7,7 & \\
\hline & 3 & 1 & 6,3 & 3 & 13,0 & 4 & 10,3 & \\
\hline \multirow{4}{*}{ L5-S1 } & 0 & 13 & 81,3 & 11 & 47,8 & 24 & 61,5 & \multirow{4}{*}{0,050} \\
\hline & 1 & 2 & 12,5 & 4 & 17,4 & 6 & 15,4 & \\
\hline & 2 & 1 & 6,3 & 5 & 21,7 & 6 & 15,4 & \\
\hline & 3 & 0 & 0,0 & 3 & 13,0 & 3 & 7,7 & \\
\hline
\end{tabular}

\begin{tabular}{|c|c|c|c|c|c|c|c|c|}
\hline \multicolumn{9}{|c|}{ Forame (esquerdo) } \\
\hline \multirow{4}{*}{ L1-L2 } & 0 & 15 & 93,8 & 19 & 86,4 & 34 & 89,5 & \multirow{4}{*}{0,693} \\
\hline & 1 & 0 & 0,0 & 1 & 4,5 & 1 & 2,6 & \\
\hline & 2 & 1 & 6,3 & 0 & 0,0 & 1 & 2,6 & \\
\hline & 3 & 0 & 0,0 & 2 & 9,1 & 2 & 5,3 & \\
\hline \multirow{3}{*}{ L2-L3 } & 0 & 15 & 93,8 & 18 & 81,8 & 33 & 86,8 & \multirow{3}{*}{0,529} \\
\hline & 1 & 1 & 6,3 & 3 & 13,6 & 4 & 10,5 & \\
\hline & 3 & 0 & 0,0 & 1 & 4,5 & 1 & 2,6 & \\
\hline \multirow{4}{*}{ L3-L4 } & 0 & 14 & 87,5 & 12 & 52,2 & 26 & 66,7 & \multirow{4}{*}{0,057} \\
\hline & 1 & 1 & 6,3 & 5 & 21,7 & 6 & 15,4 & \\
\hline & 2 & 1 & 6,3 & 4 & 17,4 & 5 & 12,8 & \\
\hline & 3 & 0 & 0,0 & 2 & 8,7 & 2 & 5,1 & \\
\hline \multirow{4}{*}{ L4-L5 } & 0 & 14 & 87,5 & 5 & 21,7 & 19 & 48,7 & \multirow{4}{*}{$<0,001$} \\
\hline & 1 & 2 & 12,5 & 7 & 30,4 & 9 & 23,1 & \\
\hline & 2 & 0 & 0,0 & 6 & 26,1 & 6 & 15,4 & \\
\hline & 3 & 0 & 0,0 & 5 & 21,7 & 5 & 12,8 & \\
\hline \multirow{4}{*}{ L5-S1 } & 0 & 13 & 81,3 & 10 & 43,5 & 23 & 59,0 & \multirow{4}{*}{0,022} \\
\hline & 1 & 2 & 12,5 & 0 & 0,0 & 2 & 5,1 & \\
\hline & 2 & 0 & 0,0 & 5 & 21,7 & 5 & 12,8 & \\
\hline & 3 & 1 & 6,3 & 8 & 34,8 & 9 & 23,1 & \\
\hline
\end{tabular}

Não houve correlação entre as medidas feitas à RM e as queixas de lombalgia, radiculopatia, claudicação ou mista. No entanto, os pacientes com queixa de radiculopatia apresentam valores menores de ASTSD nos níveis L4-L5 e L5-S1 ( $p=0,004$ e $p=0,001$ respectivamente, pela correlação de Spearman)

\section{DISCUSSÃO}

A ECL é uma condição relativamente comum em nossos dias, estando relacionada ao envelhecimento da população ${ }^{2,10,19}$. Como doença degenerativa, é incomum antes dos 50 anos de idade 20 . As comorbidades que coexistem nesta população idosa, associadas à evolução lenta da doença, tornam o diagnóstico ainda mais desafiador ${ }^{21}$. Em nosso estudo, a idade média dos grupos foi de 53,15 anos, 55 anos para o grupo Estenose, que tinha o diagnóstico. A maioria das séries mostra que a afecção predomina no sexo masculino ${ }^{22}$, porém em nosso estudo não houve predominância estatisticamente significativa.

Por não haver uma relação clara entre o grau de estreitamento do canal e os sintomas na ECL, os exames de imagem, associados aos achados clínicos, são importantes para o tratamento adequado destes pacientes ${ }^{11,20}$. O diagnóstico da estenose envolve exames de imagem e avaliação clínica, sendo esta de caráter subjetivo. Utilizamos o questionário de incapacidade Oswestry (IO), por ser de fácil compreensão, com respostas rápidas e objetivas, não exigindo qualquer treinamento ou custos elevados. A versão traduzida do questionário foi facilmente compreendida e teve uma taxa de resposta de $80 \%{ }^{17}$. Este questionário auto-administrado é reprodutível, confiável e consistente, sendo um instrumento útil para a avaliação de incapacidade em pacientes com dor lombar ${ }^{23}$.

Em nosso estudo, houve uma diferença significativa do 10 entre os grupos; observamos um 10 maior para o grupo com estenose. Contudo, não conseguimos correlacionar o grau de estenose verificado na imagem com os achados clínicos neste grupo. Vários autores também descrevem esta falta de correlação ${ }^{11,19}$, porém sem utilizar grupos controle nos estudos. Num estudo recente de Sigmundsson et al. ${ }^{10}$, foi encontrada correlação entre a área do saco dural e a presença de doença multinível, mas surpreendentemente, estenose em três níveis se associou com quadro de menos dor. Pacientes com estenose multinível tiveram nível de saúde geral mais favorável que pacientes com estenose em um só nível, de acordo com os autores. Portanto, a presença de incapacidade é, realmente, significativamente mais frequente em pacientes com ECL comparados com controles sem estenose, como mostrado em nosso estudo, mas o grau de incapacidade não está correlacionado com as medidas em RNM. Como encontrado na coorte de Sigmundsson et al. ${ }^{10}$, radiculopatia é mais frequente em pacientes com ECL, e nosso estudo pôde mostrar que é significativamente mais frequente na ECL que em controles sem estenose.

Em nossa investigação, encontramos presença de alguma incapacidade no grupo controle, conforme verificado ao preenchimento do questionário de Oswestry. Porém não foi encontrada evidência de estenose no exame de imagem desses participantes. Atribuímos tal achado à susceptibilidade individual das raízes ou a obstrução dinâmica, o que não pôde ser verificado pelo exame de RNM realizado neste estudo, já que foi realizado com o paciente estático em posição deitada. Acrescentamos também a essas possibilidades o estado emocional, pois pacientes psicologicamente comprometidos e com qualquer grau de depressão apresentam exacerbação da dor ${ }^{24-26}$. Interessante para trabalhos futuros talvez fosse realizar uma avaliação psicológica prévia dos participantes.

A lombalgia, encontrada como queixa em ambos os grupos, tem, no entanto, características diferentes conforme o grupo. No Grupo Estenose, é justificada, pois é a principal queixa na $\mathrm{ECL}^{19}$. No Grupo Controle, causas mecânicas e degenerativas sem comprometimento do canal justificam a presença, na faixa etária estudada ${ }^{27,28}$.

$\mathrm{Na}$ avaliação dos resultados da ASTSC, encontramos que os pacientes do grupo com estenose apresentam menor valor nos 
níveis L3-L4, L4-L5 e L5-S1. Estes níveis são os que apresentam maior mobilidade, estando, portanto, mais suscetíveis à degeneração ${ }^{29}$. O diâmetro do canal mostrou-se diminuído em todas as vértebras deste grupo, fato justificável, pois são pacientes selecionados para a cirurgia, já com diagnóstico de estenose.

$\mathrm{Na}$ avaliação da estenose dos recessos laterais e forames, encontramos que o contato com a raiz esteve presente em ambos os grupos, porém superior no grupo com estenose do canal. $O$ comprometimento dos forames e recesso lateral no grupo controle mostra que a RNM isoladamente não tem valor preditivo para a $\mathrm{ECL}^{30}$.

\section{CONCLUSÃO}

Não houve relação entre o grau de ECL mensurado em exame de RNM com o índice de Oswestry em ambos os grupos.

\section{REFERÊNCIAS}

1. Goh KJ, Khalifa W, Anslow P, Cadoux-Hudson T, Donaghy M. The clinical syndrome associeted with lumbar spinal stenosis. Eur Neurol. 2004;52(4):242-9.

2. Arbit E, Pannullo S. Lumbar stenosis: a clinical review. Clin Orthop Relat Res. $2001 ;(384): 137-43$

3. Zouboulis P, Karageorgos A, Dimakopoulos P, Tyllianakis M, Matzaroglou C, Lambiris E. Functional outcome of surgical treatment for multilevel lumbar spinal stenosis. Acta Orthop. 2006;77(4):670-6.

4. Szpalski M, Gunzburg R. Lumbar spinal stenosis: clinical features and new trends in surgical treatment. Geriatr Times. 2004;5(4):11. Available from: http://www.cmellc.com/ geriatrictimes/g040811.html. Accessed in 2012 (Mar 1).

5. Thomé C, Börm W, Meyer F. Degenerative lumbar spinal stenosis: current strategies in diagnosis and treatment. Dtsch Arztebl Int. 2008;105(20):373-9.

6. Postacchini F. Lumbar spinal stenosis and pseudostenosis. Definition and classification of pathology. Ital J Orthop Traumatol. 1983;9(3):339-50

7. Fritz JM, Delitto A, Welch WC, Erhard RE. Lumbar spinal stenosis: a review of current concepts in evaluation, management, and outcome measurements. Arch Phys Med Rehabil. 1998;79(6):700-8.

8. Moon ES, Kim HS, Park JO, Shin DE, Ha JW, KwakYH, et al. Comparison of the predictive value of myelography, computed tomography and MRI on the treadmill test in lumbar spinal stenosis. Yonsei Med J. 2005;46(6):806-11.

9. Schonstrom NS, Boleander NF, Spengler DM. The pathomorphology of spinal stenosis as seen on CT scans of the lumbar spine. Spine (Phila Pa 1976). 1985:10(9):806-11.

10. Sigmundsson FG, Kang XP, Jönsson $B$, Strömqvist B. Correlation between disability and MRI findings in lumbar spinal stenosis: a prospective study of 109 patients operated on by decompression. Acta Orthop. 2011:82(2):204-10.

11. Lohman CM, Tallroth K, Kettunen JA, Lindgren KA. Comparasion of radiologic signs and clinical symptoms of spinal stenosis. Spine (Phila Pa 1976). 2006;31(16):1834-40.

12. Barz T, Melloh M, Staub LP, Lord SJ, Lange J, Röder CP, et al. Nerve root sedimentation sign: evaluation of a new radiological sign in lumbar spinal stenosis. Spine (Phila Pa 1976). 2010;35(8):892-7.

13. Schizas C, Theumann N, Burn A, Tansey R, Wardlaw D, Smith FW, et al. Qualitative grading of severety of lumbar spinal stenosis based on the morphology of the dural sac on magnetic resonance images. Spine (Phila Pa 1976). 2010;35(21):1919-24.

14. Fairbank JC, Couper J, Davies JB, O'Brien JP. The Oswestry low back pain disability questionnaire. Physiotherapy. 1980;66(8):271-3.

15. Fairbank JC, Pynsent PB. The Oswestry disability index. Spine (Phila Pa 1976). 2000;25(22):2940-52.
16. Weishaupt D, Schmid MR, Zanetti M, Boos N, Romanowski B, Kissling RO, et al. Positional MR imaging of the lumbar spine: does it demonstrate nerve root compromise not visible at conventional MR imaging? Radiology. 2000;215(1):247-53.

17. Vigatto R. Adaptação cultural do instrumento "The low back pain disability oswestry questionnaire" [Cross-cultural adaptation of oswerstry disability index]. [dissertation] Campinas: Universidade Estadual de Campinas. Faculdade de Ciências Médicas; 2006.

18. Kirkwood B, Sterne J. Essential medical statistics. Blackwell Science, Philadelphia; 2006.

19. Sirvanci M, Bhatia M, Ganiyusufoglu KA, Duran C, Tezer M, Ozturk C, et al. Degenerative lumbar spinal stenosis: correlation with Oswestry Disability Index and MR imaging. Eur Spine J. 2008;17(5):679-85

20. Genevay S, Atlas SJ. Lumbar spinal stenosis. Best Pract Res Clin Rheumatol. 2010:24(2):253-65

21. Karppinen J, Shen FH, Luk KD, Andersson GB, Cheung KM, Samartzis D. Management of degenerative disk disease and chronic low back pain. Orthop Clin North Am. 2011:42(4):513-28.

22. Katz JN, Dalgas M, Stucki G, Lipson SJ. Diagnosis of lumbar spinal stenosis. Rheum Dis Clin North Am. 1994;20(2):471-83.

23. Falavigna A, Teles AR. Avaliação clínica e funcional no pré-operatório de doenças degenerativas da coluna vertebral. Coluna/Columna. 2009;8(3):245-53.

24. Campbell P, Jordan KP, Dunn KM. The role of relationship quality and perceived partner responses with pain and disability in those with back pain. Pain Med. 2012;13(2):204-14

25. Hart DL, Werneke MW, Deutscher D, George SZ, Stratford PW, Mioduski JE. Using intake and change in multiple psychosocial measures to predict functional status outcomes in people with lumbar spine syndromes: a preliminary analysis. Phys Ther. 2011;91(12):1812-25.

26. Carragee EJ, Alamin TF, Miller JL, Carragee JM. Discographic, MRI and psychosocial determinants of low back pain disability and remission: a prospective study in subjects with benign persistent back pain. Spine J. 2005;5(1):24-35

27. Apazidis A, Ricart PA, Diefenbach CM, Spivak JM. The prevalence of transitional vertebrae in the lumbar spine. Spine J. 2011;11(9):858-62.

28. Boden SD. The use of radiographic imaging studies in the evaluation of patients who have degenerative disorders of the lumbar spine. J Bone Joint Surg Am. 1996;78(1):114-24

29. Aalto TJ, Malmivaara A, Kovacs F, Herno A, Alen M, Salmi L, et al. Preoperative predictors for postoperative clinical outcome in lumbar spinal stenosis: systematic review. Spine (Phila Pa 1976). 2006;31(18):E648-63.

30. Zeifang F, Schiltenwolf M, Abel R, Moradi B. Gait analysis does not correlate with clinical and MR imaging parameters in patients with symptomatic lumbar spinal stenosis. BMC Musculoskelet Disord. 2008;9:89 